\title{
Retração de cintura escapular em recém- nascidos internados em uma Unidade de Cuidados Intermediários
}

\section{Scapular waist retraction in newborns admitted to an Intermediate Care Unit}

\author{
Josiane Silva Gomes ${ }^{1}$ (1) \\ Tatiane Falcão dos Santos Albergaria ${ }^{2}$ (1) \\ Carmen Júlia Del Rey Villa Flor ${ }^{3}$ (1) \\ Elzo Pereira Pinto Júnior ${ }^{4}$ (1) \\ lana Monteiro Lima Gomes 5 (1)
}

\author{
'Autora para correspondência. Universidade do Estado da Bahia (Salvador). Bahia, Brasil. josigomes04@hotmail.com \\ 2Universidade do Estado da Bahia (Salvador). Bahia, Brasil. tfsalbergaria@uneb.br \\ ${ }^{3}$ Hospital Geral Roberto Santos (Salvador). Bahia, Brasil. cjdelrey@hotmail.com \\ ${ }^{4}$ Centre for Data and Knowledge Integration for Health (CIDACS/ Fiocruz BA) (Salvador). Bahia, Brasil. elzojr@hotmail.com \\ ${ }^{5}$ Hospital Geral Roberto Santos (Salvador). Bahia, Brasil. ianafisio@yahoo.com.br
}

RESUMO | INTRODUÇÃo: As unidades de cuidados neonatais dispõem de altos recursos tecnológicos que, aliados ao avanço da ciência, visam garantir a sobrevida de recém-nascidos (RNs). Entretanto, o internamento expõe os RNs a variados estímulos que, associados a assistência inadequada, podem favorecer o surgimento de alterações posturais. OBJETIVO: Investigar a frequência dos sinais sugestivos de retração de cintura escapular em RNs que necessitaram de internamento. MATERIAIS E MÉTODOS: Estudo observacional, quantitativo e longitudinal, realizado com $16 \mathrm{RNs}$, internados na Unidade de Cuidados Intermediários (UCI) de um hospital estadual, localizado na cidade de cidade de Salvador, Bahia, no ano de 2019. Os dados foram coletados por meio de medidas das escápulas, sinal do cachecol, protocolo de inspeção biomecânica e prontuários. RESULTADOS: Sete $(43,7 \%)$ RNs apresentaram sinais sugestivos de retração de cintura escapular. Seis $(37,5 \%)$ apresentaram sinal do cachecol positivo e apenas um $(6,3 \%)$ apresentou simultaneamente os sinais de abdução de membros superiores, adução escapular, sinal do cachecol positivo e redução das medidas das escápulas em relação à coluna ao longo do internamento. A comparação entre a diferença do maior e do menor valor das medidas das escápulas, com o sinal do cachecol, não foi estatisticamente significante. Porém, aqueles que apresentaram sinal do cachecol positivo e/ou diminuição das medidas atingiram valores máximos de dias de internamento. CONCLUSÃO: Os sinais sugestivos de retração de cintura escapular estiveram presentes em quase metade da amostra. Importante salientar que esses achados traduzem as características específicas da população do estudo, considerando suas limitações.

PALAVRAS-CHAVE: Recém-nascido. Anormalidades

Musculoesqueléticas. Hospitalização. Escápula.
ABSTRACT | INTRODUCTION: Neonatal care units have high technological resources that, together with the advancement of science, aim to ensure the survival of newborns (NBs). However, hospitalization exposes newborns to various stimuli that, associated with inadequate care, can favor the appearance of postural changes. OBJECTIVE: To investigate the frequency of signs suggestive of scapular waist retraction in newborns who required hospitalization. Materials and METHODS: Observational, quantitative, and longitudinal study, carried out with 16 newborns, admitted to the Intermediate Care Unit (ICU) of a state hospital, located in the city of Salvador, Bahia, in the year 2019. Data were collected through scapula measurements, scarf signs, biomechanical inspection protocol, and medical records. RESULTS: Seven (43.7\%) of the newborns showed signs suggestive of scapular waist retraction. Six (37.5\%) showed a positive scarf sign and only one (6.3\%) simultaneously showed signs of the abduction of the upper limbs, scapular adduction, positive scarf sign, and reduced scapular measurements in relation to the spine along with hospitalization. The comparison between the difference between the highest and lowest scapular measurements, with the scarf sign, was not statistically significant. However, those who showed a positive scarf sign and/or reduced measurements reached maximum values for days of hospitalization. CONCLUSION: Signs suggestive of scapular waist retraction were present in almost half of the sample. It is important to note that these findings reflect the characteristics of the study population, considering their limitations.

KEYWORDS: Newborn. Musculoskeletal abnormality. Hospitalization. Scapulas. 


\section{Introdução}

As unidades de cuidados neonatais são ambientes estruturalmente preparados para atender as variadas demandas, dispondo de uma série de equipamentos de alta tecnologia, que aliados ao avanço da ciência, visam garantir a sobrevida dos recém-nascidos (RNs). Entretanto, apesar dos benefícios, a rotina das unidades hospitalares expõe os RNs a estímulos sensoriais excessivos que podem ser nocivos ao seu desenvolvimento. Nesse sentido, estes tendem a enfrentar grandes desafios para a manutenção da auto-organização e ajuste ao ambiente extra-uterino ${ }^{1 \frac{1}{2}}$.

O posicionamento é um dos procedimentos realizados nas unidades neonatais que pode exercer grande influência no desenvolvimento neuromotor dos RNs, visto que os sistemas neurológico e musculoesquelético estão em constante processo de formação nesta fase da vida ${ }^{1}$. Sendo assim, quando os RNs, principalmente os prematuros, são posicionados de forma incorreta ou mantidos em posição restrita por longos períodos, estes tendem a desenvolver alterações posturais, a exemplo da hiperextensão de tronco, que predispõe o surgimento de uma postura predominantemente fixa a retração de cintura escapular1-4. Esta pode estar associada à elevação, rotação externa e abdução dos ombros, flexão de cotovelo e hiperextensão da coluna cervical ${ }^{5,6}$. Além disso, o conjunto destas alterações podem comprometer a amplitude de movimento do complexo dos ombros/membros superiores (MMSS)'.

Dentre os mecanismos fisiológicos que podem estar envolvidos no desenvolvimento da retração de cintura escapular em RNs, destacam-se: o desequilíbrio entre a ação dos músculos flexores e extensores do tronco; a alteração transitória do tônus muscular; e a ação da gravidade contra uma musculatura neurologicamente imatura, especialmente nos recém-nascidos prematuros. O desenvolvimento de hipertonia nos músculos trapézio (porção média) e rombóides, principais adutores das escápulas, também pode contribuir para o desenvolvimento desta alteração?

A retração de cintura escapular pode acarretar limitações funcionais ao longo do desenvolvimento, pois afasta o RN da orientação a linha média, com consequente déficit na coordenação olho-mão ${ }^{8}$, atraso nas atividades exploratórias de levar a mão à boca ${ }^{9}$, mão à mão, manipular e transferir objetos, tarefas importantes no primeiro ano de vida ${ }^{10}$. Além disso, a limitação para realizar o movimento de alcance aos quatro e seis meses de vida pode estar associada à alteração na coordenação motora fina em torno dos seis anos de idade ou na fase escolar ${ }^{11}$. Neste contexto, apesar de já ser estabelecida na literatura a associação entre as alterações posturais e as interferências no desenvolvimento, poucos estudos investigaram como desfecho principal os sinais sugestivos de retração de cintura escapular. Sendo assim, é relevante o conhecimento sobre esta temática, visto que ela pode estar associada a limitações funcionais e atrasos nas aquisições motoras ao longo do desenvolvimento infantil ${ }^{2,11,12}$.

Este estudo teve como objetivo geral investigar a frequência dos sinais sugestivos de retração de cintura escapular em RNs que necessitaram de internamento em uma unidade de cuidados especializados neonatais.

\section{Materiais e métodos}

Trata-se de um estudo quantitativo, observacional e longitudinal. A população foi composta por RNs internados na Unidade de Cuidados Intermediários (UCI) de uma instituição hospitalar estadual do Sistema Único de Saúde (SUS), referência em Neonatologia, localizada na cidade de Salvador, Bahia. Foram incluídos aqueles RNs que necessitaram de internamento nas primeiras 48 horas de vida e que não apresentaram suspeita ou diagnóstico de síndrome genética, paralisia braquial ou má formação congênita. Além disso, frente ao risco de hemorragia peri-intraventricular, RNs pré-termos com idade gestacional inferior a 33 semanas não foram incluídos na amostra, considerando a necessidade de posicionamento em decúbito ventral.

O critério de exclusão do estudo foi irritabilidade sem resposta ao estímulo de sucção não nutritiva para ajuste do estado comportamental, durante a realização das medidas do posicionamento das escápulas ou sinal do cachecol. A amostragem do estudo foi por conveniência. 
O período da coleta de dados foi compreendido de julho a novembro de 2019. Visando evitar possíveis inconsistências nos resultados, as avaliações foram realizadas apenas por uma das pesquisadoras. Um estudo piloto com cinco RNs foi realizado, com o objetivo de calibrar a aplicação dos instrumentos de avaliação utilizados e os processos relacionados à coleta de dados.

Nos dias de coleta de dados, as pesquisadoras visitavam a UCl identificando aqueles RNs que cumpriam os critérios de inclusão do estudo, como também realizavam as avaliações/acompanhamento daqueles já incluídos na pesquisa. As avaliações foram realizadas três vezes na semana, iniciando no dia da admissão do RN na unidade até o dia da alta médica no setor. Os estados comportamentais de alerta ou alerta com atividade (estados quatro e cinco da escala de Brazelton, respectivamente) ${ }^{13}$ foram considerados necessários durante as coletas, pois estes são estados essenciais para se obter as respostas adequadas, visto que em estados comportamentais de sonolência ou choro pode haver alteração do tônus muscular, o que poderia interferir nos resultados obtidos ${ }^{13}$. Sendo assim, quando necessário, o estímulo à sucção não nutritiva foi realizado, para uma melhor adequação do estado comportamental.

Frente à escassez de medidas validadas na literatura que permitissem a identificação/diagnóstico dos sinais sugestivos de retração de cintura escapular, o protocolo de coleta de dados utilizou-se de mais de uma medida de avaliação. Para realizar a investigação, foi desenvolvido pelos autores do estudo um formulário estruturado contendo variáveis sobre as características demográficas e clínicas dos RNs e variáveis referentes ao internamento hospitalar. A análise da biomecânica postural foi realizada utilizando-se um protocolo de inspeção biomecânica, elaborado pelos pesquisadores do estudo, e baseado nas variáveis do estudo de Georgieff e Bembaum ${ }^{10}$ e Oliveira et al. ${ }^{14}$.

A avaliação da mobilidade do complexo cintura escapular/membro superior foi realizada por meio do sinal de cachecol, analisando a localização do olécrano em relação à linha média do tronco. Quando este sinal apresentava alteração, sugeria comprometimento da mobilidade da cintura escapular, por meio da resistência oferecida ao movimento na presença de hipertonia ou completa ausência de resistência na presença de hipotonia muscular. Para realizar o sinal do cachecol, passivamente os MMSS do RN foram levados ao movimento de flexão anterior e adução sobre o tórax, de modo que sua extremidade distal alcançasse o ombro contralateral, analisando a posição assumida pelo olécrano em relação à linha média do tronco 13,15 .

Neste estudo, a alteração do sinal do cachecol foi considerada positiva para hipertonia quando, nos RNs a termo, o olécrano não conseguiu alcançar a linha média do tronco, e nos prematuros, quando o olécrano não ultrapassou a linha média, ou seja, quando o olécrano apenas alcançou a linha média ou não conseguiu atingi-la.

A avaliação do posicionamento das escápulas foi realizada por meio de medidas em centímetros, das distâncias das bordas mediais superiores e inferiores das escápulas até a coluna vertebral̋. Estas medidas foram realizadas com os RNs em decúbito ventral elevado, sobre um posicionador (cueiro), na posição vertical, sendo o comprimento deste a distância da face (arco zigomático) até a região inferior do tronco e a largura a distância entre as linhas axilares direita e esquerda, baseando-se nos estudos de Monfort e Smith ${ }^{3}$. Após o RN ter sido posicionado, as medidas foram realizadas duas vezes durante a avaliação, as primeiras medidas foram realizadas com a cervical em rotação para a direita e as segundas medidas com a cervical em rotação para a esquerda, visando identificar se a rotação da coluna cervical exercia influência no posicionamento das escápulas.

Após a coleta dos dados, estes foram tabulados e análises estatísticas foram realizadas em duas etapas. Na primeira delas, foram utilizados conceitos da estatística descritiva para calcular as frequências absolutas e relativas (percentuais) das variáveis categóricas, além das medidas de tendência central (média ou mediana) e medidas de dispersão (desvio-padrão ou intervalos interquartílicos) das variáveis contínuas. Após a análise descritiva univariada, a análise bivariada foi utilizada para testar a associação entre o desfecho (sinais sugestivos de retração de cintura escapular) e as demais variáveis clínicas dos RNs. 
Para observar as diferenças de médias das variáveis quantitativas de acordo com a presença/ausência dos sinais sugestivos de retração de cintura escapular, medido pelo sinal do cachecol, foi calculado o Teste $T$ de Student. Em todas as análises bivariadas foram consideradas estatisticamente significantes as associações cujo valor de $p$ foi menor que 0,05 (valor de $p<0,05$ ). Os dados foram tabulados em planilhas de texto do Microsoft Excel 2013 e em seguida foi realizado um procedimento de conferência e limpeza dos dados para evitar possíveis inconsistências nos resultados, logo após, os dados foram analisados no software de análise estatística Stata, v.12. O projeto de pesquisa foi aprovado pelo Comitê de Ética em Pesquisa do hospital, pelo CAAE: 11638119.0.0000.5028.

\section{Resultados}

No período compreendido de julho a novembro de 2019, foram identificados e avaliados 16 RNs que preencheram os critérios de elegibilidade. Dentre esses RNs, 10 (62,5\%) eram do sexo masculino. Os diagnósticos clínicos mais frequentes foram prematuridade e síndrome do desconforto respiratório, observados em 11 crianças $(68,7 \%)$ (Tabela 1$)$.

Sete $(43,7 \%)$ RNs apresentaram sinais sugestivos de retração de cintura escapular. O sinal do cachecol positivo foi observado em seis $(37,5 \%)$ RNs, a abdução dos ombros e adução escapular foram observados em apenas um (6,3\%) participante (Tabela 2).

Dentre aqueles que apresentaram os sinais sugestivos, dois $(28,5 \%)$ foram pré-termo limítrofe, com 34 semanas de idade gestacional (IG), cinco $(71,4 \%)$ foram a termo, com IG maior ou igual a 37 semanas; dois $(28,5 \%)$ utilizaram acesso central (jugular direita e membro superior direito respectivamente), e um $(14,2 \%)$ foi submetido a procedimento cirúrgico (cistostomia aberta). Dos que apresentaram sinal do cachecol positivo, apenas um $(16,6 \%)$ foi prematuro. A média do período de internamento foi de 15 dias (DP $\pm 10,9$ ).
Foi observado que a rotação da coluna cervical não produziu influência sobre as medidas das bordas mediais das escápulas em relação à coluna, por este motivo, a análise das medidas apresentadas neste estudo foi relacionada à rotação da cervical para a direita. $A$ Tabela 3 apresenta as medidas das distâncias das bordas mediais das escápulas em relação à coluna dos RNs. As maiores médias de distâncias foram observadas na Borda Inferior Direita (BID) (Média=3,26; DP 0,40 ) e na Borda Inferior Esquerda (BIE) (Média=3,26; DP 0,41$)$. A diferença entre as medidas da BIE, comparando o primeiro e o último dia de internação na $U C l$, foi de 0,41 centímetros (DP 0,34).

Quando realizada a comparação entre o sinal do cachecol e as diferenças das medidas da Borda Superior Direita (BSD) e da BIE das escápulas em relação à coluna, não foram verificados valores estatisticamente significantes (Tabela 4).

A comparação da média de dias de internamento entre os RNs com presença ou ausência do sinal de cachecol, evidenciou que àqueles que apresentaram sinal do cachecol positivo tiveram uma internação mais longa, em comparação àqueles que não apresentaram (Gráfico 1).

Três participantes do estudo que foram submetidos a um maior período de internamento hospitalar evoluíram com reduções progressivas das medidas das bordas mediais das escápulas em relação à coluna. Além disso, um dos três participantes, que teve um tempo superior de internação em relação aos outros, apresentou, junto com a redução das medidas da BIE da escápula, o sinal do cachecol positivo e a abdução dos MMSS com adução das escápulas.

As demais variáveis referentes ao internamento hospitalar, como utilização de Ventilação Mecânica Invasiva (VMI), Ventilação Mecânica Não Invasiva (VNI), uso de Sedação ou de Derivação Ventrículo Peritoneal (DVP), não apresentaram influência entre os pacientes que apresentaram sinais sugestivos de retração de cintura escapular. Tal fato pode ter ocorrido devido às características dos participantes do estudo, os quais não fizeram muito uso destes dispositivos. 
Tabela 1. Características demográficas e clínicas de recém-nascidos internados em uma Unidade de Cuidados Intermediários, de um hospital público, Salvador, Bahia, 2019

\begin{tabular}{lll}
\hline & Variáveis & $\mathbf{n}(\%)$ \\
\hline Sexo & Masculino & \\
& Feminino & $10(62,5)$ \\
& & $6(37,5)$ \\
APGAR 1' & $\leq 7$ & \\
& $>7$ & $3(18,7)$ \\
& & $13(81,2)$ \\
APGAR 5' & $\leq 7$ & \\
& $>7$ & $0(0,0)$ \\
& & $16(100,0)$
\end{tabular}

\section{Diagnóstico Clínico}

$\begin{array}{ll}\text { Prematuridade } & 11(68,7) \\ \text { AIG } & 7(43,8) \\ \text { PIG } & 5(31,3) \\ \text { SDR } & 11(68,7) \\ \text { RI } & 7(43,7) \\ \text { RM } & 10(62,5) \\ \text { RTORCHS } & 5(31,2)\end{array}$

AIG = Adequado para Idade Gestacional; PIG = Pequeno para Idade Gestacional; SDR = Síndrome do Desconforto Respiratório; RI = Risco de Infecção; RM = Risco Metabólico; RTORCHS = Risco de Toxoplasmose, Rubéola, Citomegalovirus e Herpes simples.

Fonte: Dados dos autores.

Tabela 2. Sinais sugestivos de retração de cintura escapular em recém-nascidos internados em uma Unidade de Cuidados Intermediários, de um hospital público, Salvador, Bahia, 2019

\begin{tabular}{|c|c|}
\hline Variáveis & $n(\%)$ \\
\hline \multicolumn{2}{|l|}{ Sinal do cachecol } \\
\hline Sim & $6(37,5)$ \\
\hline Não & $10(62,5)$ \\
\hline \multicolumn{2}{|l|}{ Abdução dos ombros } \\
\hline Sim & $1(6,3)$ \\
\hline Não & $15(93,8)$ \\
\hline \multicolumn{2}{|l|}{ Adução escapular } \\
\hline Sim & $1(6,3)$ \\
\hline Não & $15(93,8)$ \\
\hline
\end{tabular}

Fonte: Dados dos autores. 
Tabela 3. Médias das medidas das distâncias das bordas mediais das escápulas em relação à coluna, de recém-nascidos internados em uma Unidade de Cuidados Intermediários, de um hospital público, Salvador, Bahia, 2019

\begin{tabular}{|c|c|c|c|c|}
\hline Variáveis & Média & Desvio Padrão & Mínimo & Máximo \\
\hline BSD & 2,47 & 0,44 & 1,73 & 3,40 \\
\hline BID & 3,26 & 0,40 & 2,61 & 4,05 \\
\hline Diferença BSD & 0,29 & 0,20 & 0,10 & 0,80 \\
\hline BSE & 2,47 & 0,48 & 1,76 & 3,60 \\
\hline BIE & 3,26 & 0,41 & 2,63 & 4,10 \\
\hline Diferenças BIE & 0,42 & 0,34 & 0,00 & 1,10 \\
\hline
\end{tabular}

BSD = Borda Superior Direita; BID = Borda Inferior Direita; Diferença BSD = Maior Valor da BSD - Menor Valor da BSD, na mesma criança, no período de internação; BSE = Borda Superior Esquerda; BIE = Borda Inferior Esquerda; Diferença BIE = Maior Valor da BIE - Menor Valor da BIE, na mesma criança, no período de internação.

Fonte: Dados dos autores.

Tabela 4. Comparação entre o sinal de cachecol e as diferenças das medidas das bordas superior direita e inferior esquerda das escápulas em relação à coluna, de recém-nascidos internados em uma Unidade de Cuidados Intermediários, de um hospital público, Salvador, Bahia, 2019

\begin{tabular}{llc}
\hline Sinal do Cachecol & Diferença BSD & Diferenças BIE \\
\hline Não & 0,26 & 0,48 \\
Sim & 0,35 & 0,32 \\
Valor de p & 0,414 & 0,366
\end{tabular}

Diferença BSD = Maior valor da borda superior direita - Menor valor da borda superior direita, na mesma criança, durante o período de internação; Diferença BIE = Maior valor da borda inferior esquerda - Menor valor da borda inferior esquerda, na mesma criança, no período de internação.

Fonte: Dados dos autores.

Gráfico 1. Comparação entre as variáveis sinal do cachecol e dias de internamento, de recém-nascidos internados em uma Unidade de Cuidados Intermediários de um hospital público, Salvador, Bahia, 2019

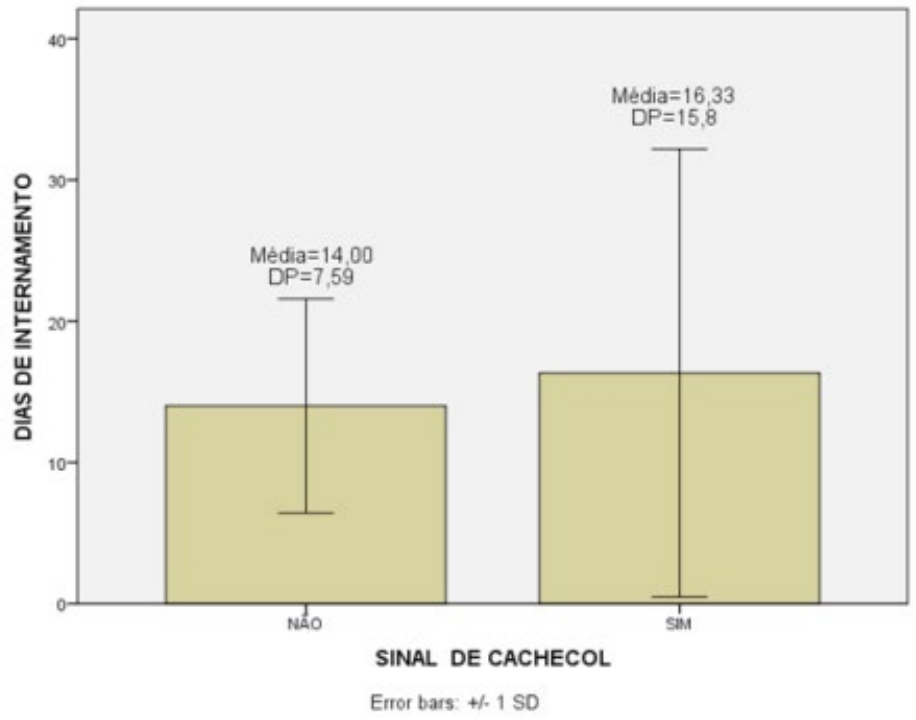

Fonte: Dados dos autores. 


\section{Discussão}

RNs internados em unidades de cuidados especiais podem apresentar sinais sugestivos de retração de cintura escapular. Nesta pesquisa, a presença destes sinais foi atribuída à anormalidade da mobilidade do complexo cintura escapular/MMSS, causada pela alteração do tônus ou encurtamento muscular, anormalidades posturais demonstradas pela presença de abdução dos ombros ou adução das escápulas e ou redução das medidas das bordas mediais das escápulas em relação à coluna durante o período de internamento até a alta hospitalar.

No presente estudo, dois RNs, um de baixo peso e outro de muito baixo peso ao nascer apresentaram sinais sugestivos de retração de cintura escapular, o primeiro obteve sinal do cachecol positivo para hipertonia, e o segundo, redução progressiva das medidas da BIE da escápula em relação à coluna, ao longo do internamento. Tal fato corrobora com os achados de Georgieff e Bembaum ${ }^{10}$, os quais descreveram em seu estudo que a incidência de retração escapular estava associada a anormalidade do tônus muscular em torno de $46 \%$ (57/125 lactentes), sendo que, destes, 74\% (42 lactentes) apresentaram retração associada à hipertonia. Além disso, estes autores verificaram que o baixo peso ao nascer, frequência de patologias respiratórias, e o maior período de internamento hospitalar, foram fatores predisponentes a adução das escápulas.

Segundo Abelha et al. $\frac{16}{}$, pacientes mais graves que necessitavam de procedimentos cirúrgicos poderiam ter uma permanência prolongada de internamento, devido ao prognóstico reservado ou a má evolução clínica, o que corrobora com os resultados deste estudo, visto que, o único paciente submetido a cirurgia permaneceu um tempo superior a 40 dias internado na UCl. Sweeney e Gutierrez $z^{5}$ descreveram que um maior período de internamento é um dos principais fatores predisponentes ao surgimento de alterações posturais, devido ao maior tempo em posições estáticas, restrições ao leito ou a insuficientes mobilizações. Os resultados deste estudo demonstraram que o maior número de dias de internamento hospitalar teve relação com os sinais sugestivos de retração escapular, concordando com os dados dos autores acima citados.

Monterosso et al. $\underline{6}$ preconizaram em seu estudo que as rotinas inadequadas de posicionamento podem favorecer uma maior ativação da musculatura extensora de tronco e, consequentemente, a retração escapular, associada à abdução dos ombros, o que pode contrariar o desenvolvimento funcional do comportamento dos MMSS a linha média. Segundo Monfort e Smith ${ }^{3}$, o risco para o desenvolvimento destas alterações posturais pode estar relacionado, principalmente, à permanência do RN em qualquer posição, por um longo período.

Segundo Sweeney e Gutierrez ${ }^{5}$, recém-nascidos que permanecem em posições restritas com pressões prolongadas nas articulações e consequente mínimo refinamento dos mecanorreceptores são mais vulneráveis à deformação esquelética, encurtamento da musculatura e redução da mobilidade articular, fatores que podem predispor a adução das escápulas. O que corrobora com os resultados obtidos, já que neonatos que obtiveram encurtamento muscular de trapézio (porção média), rombóides e peitoral, com redução da mobilidade do complexo cintura escapular/MMSS, demonstrado pelo sinal do cachecol positivo, apresentaram um dos sinais que sugere a retração escapular.

Sendo assim, pode-se verificar que o fator tempo de hospitalização não deve ser considerado o único responsável pelo surgimento de alterações posturais e sinais sugestivos de retração de cintura escapular entre os RNs. Outros fatores se associam, como o mau posicionamento 2 , restrição ao leito, prática assistencial inadequada $a^{5,6}$ e os pós-operatórios de cirurgias torácicas ou abdominais, por favorecer a posição supina, posição associada ao surgimento de retração de ombros, abdução e rotação externa dos membros superiores e membros inferiores (MMII) ${ }^{17}$. Doureta et al. $\frac{18}{}$ descreveram que a falta de posicionamentos funcionais no leito também pode favorecer a rotação externa e abdução dos quadris, podendo comprometer os movimentos dos MMII e levar a possíveis deformidades ortopédicas futuras. 
Segundo Byrne e Campbell19, a manutenção da retração escapular associada à rotação externa dos ombros pode ser prejudicial se as devidas abordagens terapêuticas não foram adotadas. Sweeney e Gutierrez ${ }^{5}$ descreveram que estas alterações posturais podem acarretar futuramente limitações para realizar alcances e rolar. Atividades como rastejar, manipular, transferir objetos e suportar o próprio peso nos antebraços em decúbito ventral também podem ser dificultadas pela presença destas alterações ${ }^{6,10}$.

Além disso, Giachetta et al. ${ }^{20}$ verificaram que o impacto do ambiente hospitalar e o tempo em que os RNs permanecem internados são possíveis fatores de risco para atraso no desenvolvimento, devido a hiperestimulações sensoriais, excesso de manuseios, intervenções dolorosas e contínuas interrupções do ciclo sono/vigília.

Importante salientar que a presença de dor entre os RNs, principalmente entre aqueles internados, submetidos a ventilação mecânica não é incomum. Um estudo que avaliou 22 RNs prematuros, com idade gestacional menor ou igual a 32 semanas, internados em uma Unidade de Terapia Intensiva Neonatal (UTIN), verificou que aqueles criticamente doentes, submetidos a VMI, apresentaram algumas respostas fisiológicas e comportamentais de dor ${ }^{21}$.

Algumas escalas que têm o objetivo de avaliar as respostas à sensação dolorosa, como a escala de ATTIA modificada ${ }^{21}$ e a escala de dor e desconforto do recém-nascido, Échelle Douleur Inconfort Nouveau-Né (EDIN) ${ }^{22}$, apresentam a hipertonia muscular dentre seus itens de classificação. Sendo assim, pode-se supor que RNs prematuros internados em estado crítico, submetidos à VMI, possuem alto risco de apresentar um quadro álgico associado 21 e uma maior vulnerabilidade de desenvolver sinais sugestivos de retração de cintura escapular, devido a presença de hipertonia ${ }^{10}$. Entretanto, a amostra do presente estudo não compreendeu estas características.

Este estudo é pioneiro, inovador na área de Fisioterapia Neonatal, sendo o primeiro registro nesta população observando alterações posturais e sua relação com medidas quantitativas, ainda que sem valor preditivo, o que torna a produção um fomento à novas pesquisas.
Espera-se que os resultados encontrados possam alertar a equipe multiprofissional atuante nas unidades neonatais sobre a prevenção ou detecção precoce de possíveis alterações posturais que possam representar riscos para a funcionalidade e atrasos no desenvolvimento, assim como incentivar uma assistência de melhor qualidade. O profissional Fisioterapeuta como integrante desta equipe deve ser capaz de realizar medidas preventivas e/ou intervenções que reduzam os impactos da hospitalização.

As limitações presentes nesta pesquisa referem-se às dificuldades metodológicas de medida das escápulas, pois não foi identificado na literatura um instrumento de medida validado ou um valor referencial do limite de normalidade para essa alteração, o que dificultou a realização das avaliações, principalmente pelo fato de o estudo ser composto por uma população heterogênea em relação à idade gestacional, ganho ponderal, estatura e desenvolvimento do tônus muscular. Além disso, o estudo contou com um pequeno número amostral, o que limitou o poder estatístico.

Sendo assim, futuros estudos que abordem o desenvolvimento de ferramentas validadas para a identificação da retração de cintura escapular se faz de grande importância, pos a identificação precoce de fatores que podem causar atrasos no desenvolvimento é fundamental para a prevenção e ou tratamento, visando um desenvolvimento adequado.

\section{Conclusão}

O presente estudo demonstrou que os sinais sugestivos de retração de cintura escapular estiveram presentes em quase metade da amostra. Não foi encontrada correlação entre o sinal do cachecol positivo e as diferenças das medidas da BSD e da BIE das escápulas em relação à coluna. Mas aqueles RNs que apresentaram sinal do cachecol positivo para redução da mobilidade do complexo cintura escapular/ MMSS, e ou redução das medidas da BIE da escápula em relação à coluna, apresentaram uma maior variação de dias de internamento, no que diz respeito aos valores máximos alcançados. Característica essa, que foi evidenciada no participante que apresentou simultaneamente o conjunto de sinais sugestivos de 
retração de cintura escapular, sinal do cachecol positivo, abdução dos ombros, adução escapular e redução das medidas da BIE da escápula. Importante salientar que esses achados traduzem as características da população específica do estudo, considerando suas limitações.

\section{Contribuições dos autores}

Albergaria TFS participou da concepção, delineamento, buscas, coleta de dados, interpretação dos resultados, redação da pesquisa, revisão, contribuição para a versão final e encaminhamento do artigo científico. Gomes IML participou da concepção da pesquisa, auxiliou na coleta de dados e na revisão. Villa Flor CJDR auxiliou na concepção, coleta de dados, revisão e na contribuição para a versão final. Pinto Júnior EP participou da concepção, análise estatística dos dados da pesquisa, interpretação dos resultados, revisão e contribuiu para a versão final. Gomes JS participou da concepção, delineamento, buscas, coleta de dados, interpretação dos resultados, redação da pesquisa, revisão e contribuições para a versão final do artigo científico.

\section{Conflitos de interesses}

Nenhum conflito financeiro, legal ou político envolvendo terceiros (governo, empresas e fundações privadas, etc.) foi declarado para nenhum aspecto do trabalho submetido (incluindo, mas não se limitando a subvenções e financiamentos, participação em conselho consultivo, desenho de estudo, preparação de manuscrito, análise estatística, etc.).

\section{Referências}

1. Toso BRGO, Viera CS, Valter JM, Delatore S, Barreto GMS. Validação de protocolo de posicionamento de recém-nascido em Unidade de Terapia Intensiva. Rev. Bras. Enferm. 2015;68(6):83541. https://doi.org/10.1590/0034-7167.2015680621i

2. Albuquerque TM, Albuquerque RC. Estratégias de posicionamento e contenção de recém-nascido pré-termo utilizadas em unidades de terapia intensiva neonatal. Rev. Interinst. Bras. Ter. Ocup. 2017;1(1):40-51. https://doi. org/10.47222/2526-3544.rbto4254

3. Monfort K, Case-Smith J. The Effects of a Neonatal Positioner on Scapular Rotation. Am J Occup Ther. 1997;51(5):378-384. https:// doi.org/10.5014/ajot.51.5.378
4. Marta CB, Bragança KL, Leite JL, Francisco MTR, Seabra Junior $\mathrm{HC}$, Silva PO. Os posicionamentos dos recém-nascidos no leito como prática assistencial da equipe de enfermagem. Rev. Pesq. Cuid. Fundam. Online [Internet]. 2012;3(2):2521-28. Disponível em: http://www.seer.unirio.br/index.php/cuidadofundamental/ article/download/1573/pdf_585

5. Sweeney JK, Gutierrez T. Musculoskeletal implications of preterm infant positioning in the NICU. Perinat Neonat. Nurs. 2002;16(1):58-70. https://doi.org/10.1097/00005237-200206000$\underline{00007}$

6. Monterosso L, Kristjanson LJ, Cole J, Evans SF. Effect of postural supports on neuromotor function in very preterm infants to term equivalent age. Pediatr. Child Health. 2003;39(3);197-205. https:// doi.org/10.1046/j.1440-1754.2003.00125.x

7. Lessa NMC. Avaliação do desenvolvimento postural e do desempenho motor em crianças de 4 a 6 anos de idade nascidas prematuras: Visão fisioterapêutica [dissertação] [Internet]. Rio de Janeiro: Instituto Fernandes Figueira, Fundação Oswaldo Cruz. Curso de fisioterapia; 2006. Disponível em: https://www.arca. fiocruz.br/handle/icict/3495

8. Hall SJ. Biomecânica Básica. $4^{a}$. ed. Rio de Janeiro: Guanabara Koogan; 2005.

9. Prado C, Vale LA. Fisioterapia Neonatal e Pediátrica. São Paulo: Manole; 2012.

10. Georgieff MK, Bembaum JC. Abnormal shoulder girdle muscle tone in premature infants during their first 18 months of life. Pediatr. Res. 1986;77(5):664-9. Citado em: PMID: 3703633

11. Fallang B, Oien I, Hellem E, Saugstad OD, Algra MH. Quality of reaching and postural control in young preterm infants is related to neuromotor outcome at 6 years. Pediatr. Res. 2005;58:347-53. https://doi.org/10.1203/01.pdr.0000170898.60160.09

12. Moreira AM. A intervenção precoce em recém-nascidos pré-termo. O Posicionamento, a correcção postural e neuromuscular [dissertação] [Internet]. Porto: Universidade do Porto; 2004. Disponível em: https://repositorio-aberto.up.pt/ bitstream/10216/23342/2/29757.pdf

13. Amiel-Tison C. A method for neurologic evaluation within the first year of life. University of Paris. Curr Prob Pediatr 1976;7(1):150. Citado em: PMID: 975863

14. Oliveira MP, Melo MFA, Sá SMP. Retração Escapular em Rn Prematuros Sob Ventilação Mecânica Invasiva: Relato de Caso. Anais da Mcc [Internet]. 2012;1(3). Disponível em: https://www. bahiana.edu.br/revistamcc/hp/artigo.aspx?id=6283 
15. Lekskulchai R, Cole J. Scarf ratio: A method of measuring the Scarf sign in preterm born infants. Australian Journal of Physiotherapy. 2000;46(2):85-90. https://doi.org/10.1016/500049514(14)60316-0

16. Abelha FJ, Castro MA, Landeiro NM, Neves AM, Santos CC. Mortality and Length of Stay in a Surgical Intensive Care Unit. Rev Bras Anestesiol; 2006;56(1):34-45. https://doi.org/10.1590/s003470942006000100005

17. Almeida SKA, Mejia DPM. Os benefícios da estimulação motora em recém-nascidos na UTIN: Exercícios e posicionamento terapêuticos [dissertação] [Internet]. Goiás: Faculdade Sul Americana; 2014. Disponível em: https://portalbiocursos.com.br/ ohs/data/docs/26/08__Os_benefYcios_da_estimulaYYo_motora_ em recYm-nascidos na UTI.pdf

18. Vaivre-Douret L, Ennourib K, Jrad I, Garrec C, Papiernik E. Effect of positioning on the incidence of abnormalities of muscle tone in low-risk, preterm infants. European Journal of Paediatric Neurology. 2004;8(1):21-34. https://doi.org/10.1016/j. ejpn.2003.10.001
19. Byrne E, Campbell SK. Physical Therapy Observation and Assessment in the Neonatal Intensive Care Unit. Physical e Occupational Therapy in Pediatrics. 2013;33(1):39-74. https://doi. org/10.3109/01942638.2012.754827

20. Giachetta L, Nicolau CM, Costa APBM, Zuana AD. Influência do tempo de hospitalização sobre o desenvolvimento neuromotor de recém-nascidos pré-termo. Fisioter. Pesqui; 2010;17(1):24-29. https://doi.org/10.1590/S1809-29502010000100005

21. Guinsburg R, Kopelman BI, Almeida MF, Miyoshi MH. Pain in intubated and ventilated preterm neonate: multidimensional assessment and response to fentanyl analgesia. J. Pediatr. 1994; 70(2):82-90. http://dx.doi.org/10.2223/JPED.727

22. Balda RCX, Guinsburg R. A linguagem da dor no recémnascido. Departamento de Neonatologia da Sociedade Brasileira de Pediatria [Internet]. 2018. Disponível em: https://www.sbp. com.br/fileadmin/user upload/DocCient-Neonatol-Linguagem da_Dor_atualizDEz18.pdf 